\title{
InCoB2014: Systems Biology update from the Asia-Pacific
}

\author{
Shoba Ranganathan ${ }^{*}$, Tin Wee Tan², Christian Schönbach ${ }^{3,4}$ \\ From Asia Pacific Bioinformatics Network (APBioNet) Thirteenth International Conference on Bioinformatics \\ (InCoB2014) \\ Sydney, Australia. 31 July - 2 August 2014
}

\begin{abstract}
Selected papers from the 13th International Conference on Bioinformatics (InCoB2014), July 31-2 August, 2014 in Sydney, Australia have been compiled in this supplement. These range from network analysis and gene regulatory networks to systems level biological analysis, providing the 2014 update to InCoB's computational systems biology research.
\end{abstract}

\section{Introduction}

Sydney, Australia hosted the 13th InCoB (International Conference on Bioinformatics), the official conference of the Asia-Pacific Bioinformatics Network (APBioNet) [1]. Since 2006, the best InCoB papers have been published as supplements in BMC Bioinformatics, with an additional supplement in BMC Genomics since 2009. With rising interest in systems biology and holistic analysis of biological data, InCoB published its first BMC Systems Biology supplement last year [2]. This introduction provides an update to the 2014 research in systems biology from the APBioNet community.

\section{Manuscript submission and review}

Authors were offered the BMC track (supplement issues of BMC Bioinformatics, BMC Systems Biology or BMC Genomics) and PeerJ [3]. The details of acceptance rates and the reviewing process are available from our BMC Genomics overview [4]. Eleven articles with a "systems" theme briefly described in the following sections.

\section{Network analysis}

The complexity of biological systems of often represented in terms of networks where biomolecules that interact

\footnotetext{
* Correspondence: shoba.ranganathan@mq.edu.au

'Department of Chemistry and Biomolecular Sciences and ARC Centre of Excellence in Bioinformatics, Macquarie University, Sydney NSW 2109, Australia

Full list of author information is available at the end of the article
}

with each other are represented as interaction partners. Characterizing a complex biological network by measuring its topology using 11 parameters and identifying nodes and sub-networks, is computationally challenging. Chin et al. [5] have developed cytoHubba, to analyse biological networks rapidly, rank biomolecules based on their importance and provide network visualization. This tool has been constantly improved and updated since 2009 and has been used to identify networks associated with cancer metabolism, innate immunity and even complex biofilm communities. As biological networks are dynamic, Patil and Nakai [6] have developed TimeXNet to identify temporally significant sub-networks from cellular responses to stimuli. Crosstalk between different cellular compartments is essential for for cellular communication and function. Gong and Feng [7] have analysed the ER-Golgi network and identified specific genes involved in the progress of diseases such as cancer and Alzheimer's disease. Barteret al. [8] have compared networks from two different cancers, to identify disease-specific biomarkers. Regulatory networks depend on relaying signals in a cascade, resulting in time delayed circuitry, which can be analysed with high levels of sensitivity with the novel method proposed by Chen et al. [9]. For systems and synthetic biology applications, a deep understanding of the complex flux in metabolic circuits is essential. Moriya et al. [10] have approached this problem by designing a synthetic circuit, as a model system for monitoring metabolic flux. 


\section{Regulatory networks}

Understanding transcriptional regulation of genes remains a challenging problem, dependent on the binding of several transcription factors as well as epigenetic changes. Given the sparseness of experimental genomewide epigenetic profiles, Yang et al. [11] have developed the cisMEP database comprising predicted cis-regulatory modules integrated with available epigenetic data, as a first step to support research in this area. Approaching the transcription regulation problem from another angle, Yan and Wang [12] propose a new graph theoretical method to predict DNA binding sites from protein structures.

\section{Systems analysis}

A comprehensive understanding of the symbiosis between the human microbiome and the host organism is essential for defining its role in human health and disease. Yang et al. [13] have applied an ensemble clustering framework to delineate the structure of human microbiome and provide a new insight to the pathological role of microbes within the host organism. Srihari et al. [14] have analysed complexes in core cellular processes to decipher cancer mechanisms, by data integration at the protein-protein interaction and gene expression levels, across all cancer conditions.

\section{Conclusion}

The articles in this supplement span network analysis, regulatory networks as well as systems-level analysis. With ongoing NIH Big Data to Knowledge (DB2K) [15] and other similar global initiatives, we expect to see more extensive computational studies at our 2015 InCoB meeting to be held jointly with the Genome Informatics Workshop (GIW) in Tokyo, Japan [16].

\section{Competing interests}

The authors declare that they have no competing interests.

\section{Authors' contributions}

SR wrote the introduction. CS and SR (Program Committee Co-chairs) managed the review and editorial processes, respectively. TWT supported the post-acceptance manuscript processing.

\section{Acknowledgements}

We are indebted to all members of the Program Committee and additional reviewers for their efforts and time. We are grateful to the NSW T\&l Conference grant awarded to SR for hosting InCoB2014, our sponsors: the Australian Bioinformatics Network, the International Society for Computational Biology, Qiagen, Millennium Science and ABSciEx, and for material support from Macquarie University and Sydney Business Events. Special thanks go to ASN Events for running the conference smoothly. Finally, we are deeply grateful to Isobel Peters and Jennifer Egar of BMC who have supported us through the supplement publication process. This article has been published as part of BMC Systems Biology Volume 8 Supplement 4, 2014: Thirteenth International Conference on Bioinformatics (InCoB2014): Systems Biology. The full contents of the supplement are available online at http://www.biomedcentral.com/bmcsystbiol/supplements/ $8 / 54$.

\section{Authors' details}

'Department of Chemistry and Biomolecular Sciences and ARC Centre of Excellence in Bioinformatics, Macquarie University, Sydney NSW 2109, Australia. ${ }^{2}$ Department of Biochemistry, Yong Loo Lin School of Medicine, National University of Singapore, Singapore 117599. ${ }^{3}$ Department of Biology, School of Science and Technology, Nazarbayev University, Astana 010000, Republic of Kazakhstan. ${ }^{4}$ Center for AIDS Research, Kumamoto University, Kumamoto 860-0811, Japan.

\section{Published: 8 December 2014}

\section{References}

1. The Asia-Pacific Bioinformatics Network. [http://www.apbionet.org].

2. Schönbach C, Shen B, Tan T, Ranganathan S: InCoB2013 introduces Systems Biology as a major conference theme. BMC Syst Biol 2013, 7(Suppl 3):S1.

3. PeerJ. [http://peerj.com].

4. Schönbach C, Tan TW, Ranganathan S: Genomics 2014, 15(Suppl 9):11.

5. Chin $\mathrm{CH}$, Chen $\mathrm{SH}, \mathrm{Wu} H \mathrm{H}, \mathrm{Ho} \mathrm{CW}$, Ko MT, Lin CY: cytoHubba: Identifying hub objects and sub-networks from complex interactome. BMC Systems Biology 2014, 8(Suppl 4):S11.

6. Patil A, Nakai K: TimeXNet: Identifying active gene sub-networks using time-course gene expression profile. BMC Systems Biology 2014, 8(Suppl 4):S2.

7. Gong H, Feng L: Computational analysis of the roles of ER-Golgi network in the cell cycle. BMC Systems Biology 2014, 8(Suppl 4):S3.

8. Barter RL, Schramm SJ, Mann GJ, Yang YH: Network-based biomarkers enhance classical approaches to prognostic gene expression signatures. BMC Systems Biology 2014, 8(Suppl 4):S5.

9. Chen $H$, Mundra PA, Zhao LN, Lin F, Zheng J: Highly sensitive inference of time-delayed gene regulation by network deconvolution. BMC Systems Biology 2014, 8(Suppl 4):S6.

10. Moriya T, Yamamura M, Kiga D: Effects of downstream genes on synthetic genetic circuits. BMC Systems Biology 2014, 8(Suppl 4):S4.

11. Yang TH, Wang CC, Hung PC, Wu WS: cisMEP: an integrated repository of genomic epigenetic profiles and cis-regulatory modules in Drosophila. BMC Systems Biology 2014, 8(Suppl 4):S8.

12. Yan C, Wang Y: A graph kernel method for DNA-binding site prediction. BMC Systems Biology 2014, 8(Suppl 4):S10.

13. Lai FJ, Chang HT, Huang YM, Wu WS: A comprehensive performance evaluation on the prediction results of existing cooperative transcription factors identification algorithms. BMC Systems Biology 2014, 8(Suppl 4):S9.

14. Yang P, Su X, Ou-Yang L, Chua HN, Li XL, Ning K: Microbial community pattern detection in human body habitats via ensemble clustering framework. BMC Systems Biology 2014, 8(Suppl 4):S7.

15. Srihari S, Madhamshettiwar PB, Song S, Liu C, Simpson T, Khanna KK, Ragan MA: Complex-based analysis of dysregulated cellular processes in cancer. BMC Systems Biology 2014, 8(Suppl 4):S1.

16. Big Data to Knowledge (BD2K) initiative. [http://bd2k.nih.gov/].

17. GIW-InCoB. 2015 [http://incob.apbionet.org/incob15].

doi:10.1186/1752-0509-8-S4-I1

Cite this article as: Ranganathan et al:: InCoB2014: Systems Biology update from the Asia-Pacific. BMC Systems Biology 2014 8(Suppl 4):11. 